\title{
Clinical tolerance of corticospinal tracts in convection-enhanced delivery to the brainstem
}

\author{
Peter F. Morgenstern, MD, ${ }^{1,2}$ Zhiping Zhou, PhD, ${ }^{3}$ Eva Wembacher-Schröder, BS, ${ }^{4}$ \\ Vincent Cina, BS, ${ }^{4}$ Apostolos John Tsiouris, MD, ${ }^{5}$ and Mark M. Souweidane, MD ${ }^{1,2}$ \\ Departments of ${ }^{1}$ Neurological Surgery and ${ }^{5}$ Radiology, NewYork-Presbyterian Hospital, Weill Cornell Medicine, New York; \\ Departments of ${ }^{2}$ Neurosurgery and ${ }^{3}$ Pediatrics, Memorial Sloan Kettering Cancer Center, New York, New York; and ${ }^{4}$ BrainLAB, \\ Munich, Germany
}

OBJECTIVE Convection-enhanced delivery (CED) has been explored as a therapeutic strategy for diffuse intrinsic pontine glioma (DIPG). Variables that may affect tolerance include infusate volume, infusion rate, catheter trajectory, and target position. Supratentorial approaches for catheter placement and infusate distribution patterns may conflict with corticospinal tracts (CSTs). The clinical relevance of these anatomical constraints has not been described. The authors report their experience using CED in the brainstem as it relates to anatomical CST conflict and association with clinical tolerance.

METHODS In a phase I clinical trial of CED for DIPG (clinical trial registration no. NCT01502917, clinicaltrials.gov), a flexible infusion catheter was placed with MRI guidance for infusion of ${ }^{124} \mathrm{I}-8 \mathrm{H} 9$, a radioimmunotherapeutic agent. Intraand postprocedural MR images were analyzed to identify catheter trajectories and changes in T2-weighted signal intensity to approximate volume of distribution (Vd). Intersection of CST by the catheter and overlap between Vd and CST were recorded and their correlation with motor deficits was evaluated.

RESULTS Thirty-one patients with a mean age of 7.6 years (range 3.2-18 years) underwent 39 catheter insertions for CED between 2012 and 2017. Thirty catheter insertions had tractography data available for analysis. The mean trajectory length was $105.5 \mathrm{~mm}$ (range 92.7-121.6 mm). The mean number of intersections of CST by catheter was 2.2 (range $0-3$ ) and the mean intersecting length was $18.9 \mathrm{~mm}$ (range 0-44.2 mm). The first 9 infusions in the highest dose level (range 3.84-4.54 ml infusate) were analyzed for Vd overlap with CST. In this group, the mean age was 7.6 years (range 5.8-10.3 years), the mean trajectory length was $109.5 \mathrm{~mm}$ (range 102.6-122.3 mm), and the mean overlap between Vd and CST was $5.5 \mathrm{~cm}^{3}$. For catheter placement-related adverse events, 1 patient (3\%) had worsening of a contralateral facial nerve palsy following the procedure with two CST intersections, an intersecting distance of $31.7 \mathrm{~mm}$, and an overlap between Vd and CST of $3.64 \mathrm{~cm}^{3}$. For infusion-related adverse events, transient postinfusion deficits were noted in 3 patients in the highest dose level, with a mean number of 2 intersections of CST by catheter, mean intersecting length of $12.9 \mathrm{~mm}$, and mean overlap between $\mathrm{Vd}$ and CST of $6.3 \mathrm{~cm}^{3}$.

CONCLUSIONS A supratentorial approach to the brainstem crossing the CST resulted in one worsened neurological deficit. There does not appear to be a significant risk requiring avoidance of dominant motor fiber tracts with catheter trajectory planning. There was no correlation between Vd-CST overlap and neurological adverse events in this cohort. Clinical trial registration no.: NCT01502917 (clinicaltrials.gov)

https://thejns.org/doi/abs/10.3171/2018.6.JNS18854

KEYWORDS convection-enhanced delivery; diffuse intrinsic pontine glioma; corticospinal tract; intraoperative MRI; oncology

ABBREVIATIONS CED = convection-enhanced delivery; $C S T=$ corticospinal tract; $C T C A E=$ Common Terminology Criteria for Adverse Events; DIPG = diffuse intrinsic pontine glioma; $F A$ = fractional anisotropy; FOV = field of view; ROI = region of interest; Vd = volume of distribution; $\mathrm{XBRT}$ = external beam radiation therapy. SUBMITTED March 30, 2018. ACCEPTED June 20, 2018.

INCLUDE WHEN CITING Published online December 21, 2018; DOI: 10.3171/2018.6.JNS18854. 
I $\mathrm{N}$ the absence of effective therapies for diffuse intrinsic pontine glioma (DIPG), new approaches to therapeutic delivery have been explored to maximize the efficacy of investigational agents. Convection-enhanced delivery (CED) is one strategy for bypassing the bloodbrain barrier. Catheter positioning and accuracy for CED of therapeutics in DIPG have been investigated in small series. ${ }^{3}$ However, little is currently known about the tolerance of corticofugal motor fibers to catheter placement and subsequent infusion. In particular, a frontal approach to the brainstem risks traversing descending corticospinal tracts (CSTs). In addition, infusate distribution patterns are likely to overlap with corticofugal motor fibers in the pons. We have undertaken a phase I clinical trial of CED delivering ${ }^{124} \mathrm{I}-8 \mathrm{H} 9$ for DIPG patients with nonprogressive disease after external beam radiation therapy (XBRT; ClinicalTrials.gov identifier: NCT01502917). As part of this trial, we evaluated the effect of catheter placement and infusion on CST functions assessed by clinical neurological examination and report these results.

\section{Methods \\ Patients}

Eligibility criteria for this phase I clinical trial included a clinical and radiological diagnosis of DIPG, age between 3 and 21 years, prior XBRT within 4-14 weeks, no progression of tumor, and Lansky or Karnofsky Performance Scale status $\geq 50$.

\section{Pre- and Postprocedural Assessments}

Preoperative evaluation included formal neurological assessment and MRI of the brain within 14 days prior to surgery. Postoperative assessments were performed on postoperative day 1 after removal of the infusion catheter, as well as on postoperative days 4, 7, 21, and 30. Assessments on postoperative days 1 and 30 included MRI of the brain, in addition to formal neurological assessments on all scheduled postoperative evaluations. Full-body and head PET/CT scans were performed within 1-6 hours, and then at $48 \pm 24$ hours, $96 \pm 24$ hours, and $7 \pm 1$ days after completion of infusion, with up to two optional scans between days 7 and 14 .

Adverse events were classified according to the National Cancer Institute Common Terminology Criteria for Adverse Events (CTCAE; version 4, NIH Publication No. 09-5410) based on assessments performed within the first 30 days following infusion.

\section{MRI}

MRI included the following sequences: pre-gadolinium isotropic 3D T1-weighted gradient echo (slice thickness 1 $\mathrm{mm}$, matrix $256 \times 256$, slice spacing $0.5 \mathrm{~mm}$, field of view [FOV] $25 \mathrm{~cm}$ ); 2D axial T2-weighted (slice thickness 3 mm, matrix $512 \times 256$, slice spacing $3 \mathrm{~mm}$, FOV $20 \mathrm{~cm}$ ); 2D axial T2-weighted FLAIR (slice thickness $3 \mathrm{~mm}$, matrix $320 \times 224$, slice spacing $3 \mathrm{~mm}$, FOV $20 \mathrm{~cm}$ ); 3D axial T2 star-weighted angiography (slice thickness $1 \mathrm{~mm}$, matrix $320 \times 224$, slice spacing $0.5 \mathrm{~mm}$, FOV $20 \mathrm{~cm}$ ); postgadolinium 3D T1-weighted gradient echo (slice thickness $1 \mathrm{~mm}$, matrix $256 \times 256$, slice spacing $0.5 \mathrm{~mm}$, FOV 22 cm); and postgadolinium 2D axial T1-weighted FLAIR (slice thickness $3 \mathrm{~mm}$, matrix $288 \times 192$, slice spacing 3 $\mathrm{mm}$, FOV $20 \mathrm{~cm})$. Diffusion-tensor images were acquired using an echo-planar imaging sequence (35 directions, $128 \times 128$ matrix [zipped $\times 2$ ], 2-mm slice thickness, with $B$ values of 0 and $1000 \mathrm{~mm}^{2} / \mathrm{sec}$ ).

\section{Procedures}

Surgical planning for catheter (BrainLAB Flexible Catheter) placement was performed using iPlan Flow (BrainLAB), an FDA-approved software program designed to support the planning of intracranial drug delivery. The surgical target and catheter tract were planned with avoidance of sulci, ventricles, and cisterns. Stereotactic placement of the catheter was accomplished using the ClearPoint system (MRI Interventions, Inc.), an MRIcompatible skull-mounted guidance device, with an intraoperative 1.5-T MR machine (Siemens AG).

\section{Imaging Analysis}

Intraoperative postplacement MRI was fused to the pre- and postoperative image sets to assess tip accuracy using iPlan Flow. The actual tip of the catheter was extrapolated $18 \mathrm{~mm}$ from the MRI-visible ceramic-coated shaft of the catheter. Images were imported into iPlan Flow and merged using the automatic image fusion function. Apparent diffusion coefficient and fractional anisotropy (FA) maps were calculated from the diffusion data with eddy current correction.

Tractography was performed on the preoperative image set using iPlan Flow with an FA threshold of 0.20 and minimum length of $60 \mathrm{~mm}$. The values remained constant for each patient. The CST was tracked on the side of catheter insertion using two regions of interest (ROIs), one at the level of the medullary pyramid and the other over the primary motor cortex. ROIs were defined by a neurosurgeon (P.F.M.) and verified by a board-certified radiologist with a certificate of added qualification in neuroradiology (A.J.T.).

The 30 infusions performed with tractography data available were used to analyze the tolerance of CST to catheter placement. Tolerance to infusate distribution patterns was assessed in a subset of patients $(n=9)$ treated on the same dose level (highest dose level with 3.84-4.54 $\mathrm{ml}$ infusate) in order to control for infusate volume as a confounder.

\section{Intersection of CST by Catheter Trajectory}

To determine the relationship between the intersection of CST by catheter trajectory and motor deficits after catheter placement, the total length of each trajectory intersecting the fiber bundle was quantitatively examined. Trajectories were verified in probe view to see the entire course. The pan and recenter functionality in iPlan Flow was used to scroll along the trajectory and to measure the distance from the probe view plane to the target point (Fig. 1). The breakdown when the trajectories passed in and out of the tract was measured and the individual lengths were added to produce the total length of intersection.

\section{Determination of Distribution Volume}

Differences in T2-weighted images acquired at base- 

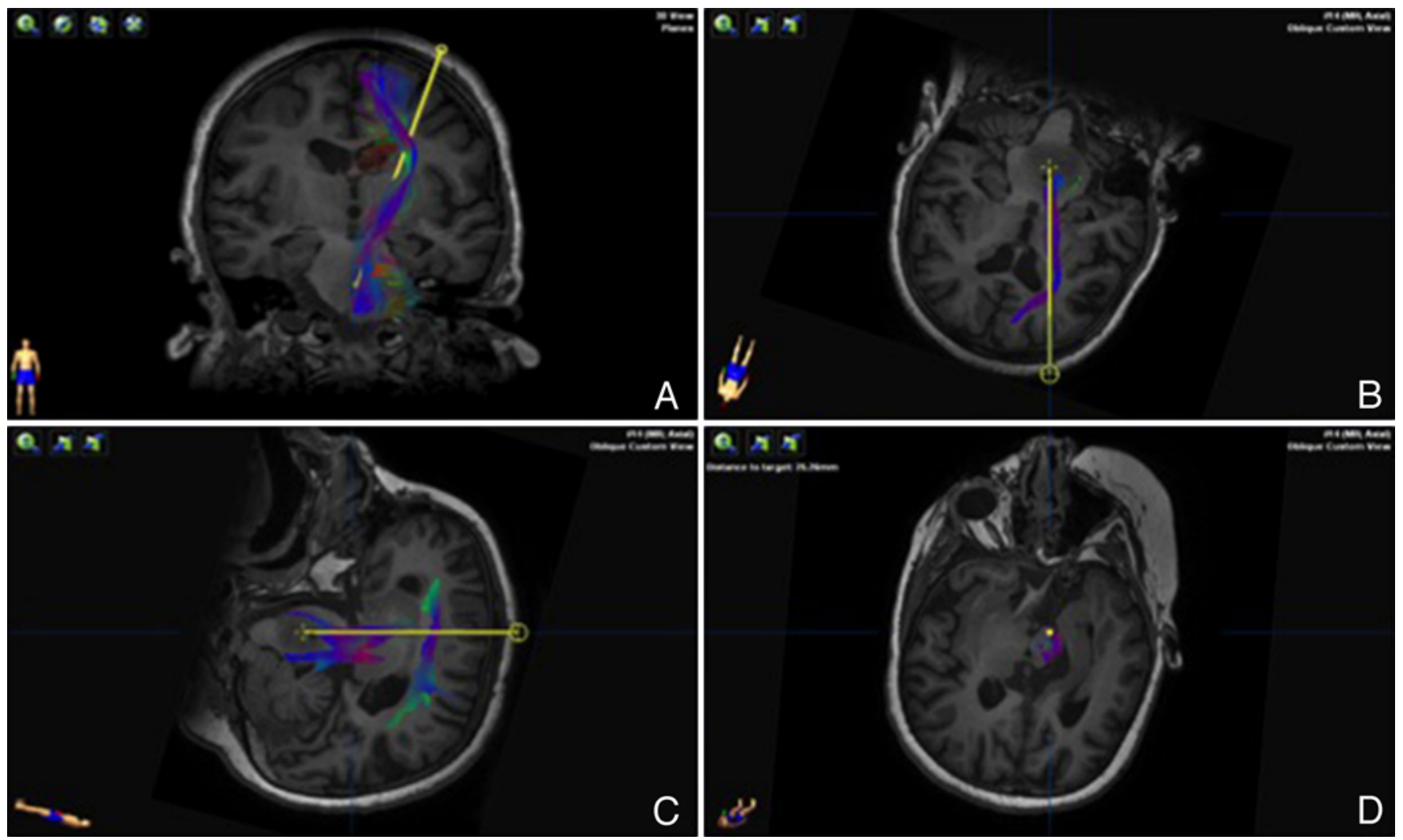

FIG. 1. Volumetric T1-weighted MRI demonstrating catheter position (yellow) and left descending CST (multicolor) in 3D projection view $(\mathbf{A})$ and in line with the catheter (B and $\mathbf{C}$ ) for a patient undergoing left frontal placement of an infusion catheter. The length of catheter intersection of the CST is identified in the probe view (D), where the frames in which the catheter traverses the CST are totaled to attain a total intersecting distance.

line and after conclusion of infusion were used to determine volume of distribution $(\mathrm{Vd})$. The area of increased T2-weighted signal intensity after ${ }^{124} \mathrm{I}-8 \mathrm{H} 9$ infusion was segmented using a semiautomatic seed-growing algorithm incorporated in iPlan Flow. In all 9 patients, PET scans obtained after conclusion of ${ }^{124} \mathrm{I}-8 \mathrm{H} 9$ infusion were coregistered to MR images.

\section{Overlap Between Vd and CST}

To determine the relationship between Vd-CST overlap and motor deficits after catheter placement and ${ }^{124} \mathrm{I}-8 \mathrm{H} 9$ infusion, the overlap volume between $\mathrm{Vd}$ and 3D models of the fiber bundle was quantitatively examined using the object manipulation function in iPlan Flow (Fig. 2).

\section{Statistical Analysis}

Grades of adverse events were treated as ordinal data. The correlation between grade of neurological deficits and number of intersections/length of intersection/overlap volume was evaluated with Spearman's correlation test. The analysis was performed in R (version 3.50, The R Foundation for Statistical Computing).

\section{Results}

Thirty-one patients with a mean age of 7.6 years (range
3.2-18 years) underwent 39 catheter insertions for CED between 2012 and 2017. Of these 39 catheter insertions, 30 had tractography data available for analysis (Table 1). In these patients, the mean trajectory length was $105.5 \mathrm{~mm}$ (range 92.7-121.6 mm). The mean number of intersections of CSTs by catheter was 2.2 (range 0-3) and the mean length of intersection was $18.9 \mathrm{~mm}$ (range 0-44.2 $\mathrm{mm}$ ).

Nine infusions were completed at the highest dose level (range 3.84-4.54 $\mathrm{ml}$ infusate) and analyzed for overlap between Vd and CST (Table 2). The mean age in this group was 7.6 years (range 5.8-10.3 years). The mean trajectory length was $109.5 \mathrm{~mm}$ (range 102.6-122.3 mm). Vd was determined in these 9 patients. In 4 of 9 patients, a clear demarcation was easily detectable throughout the entire distribution volume. In the other 5 patients, pre-infusion T2-weighted signal abnormality existed and more adjustment was required to outline the distribution volume. The congruence of the shapes of distribution determined on T2-weighted images and on PET is shown in Fig. 3. The mean Vd was $14.53 \mathrm{~cm}^{3}$. The mean overlap between $\mathrm{Vd}$ and CST was $5.5 \mathrm{~cm}^{3}$ (range $0.8-10.8 \mathrm{~cm}^{3}$ ).

One patient $(3 \%)$ had a worsened contralateral facial nerve palsy following catheter insertion that was not related to infusion and did not improve on follow-up. For this patient, there were two catheter-CST intersections with a total length of intersection of $31.7 \mathrm{~mm}$. This patient was 

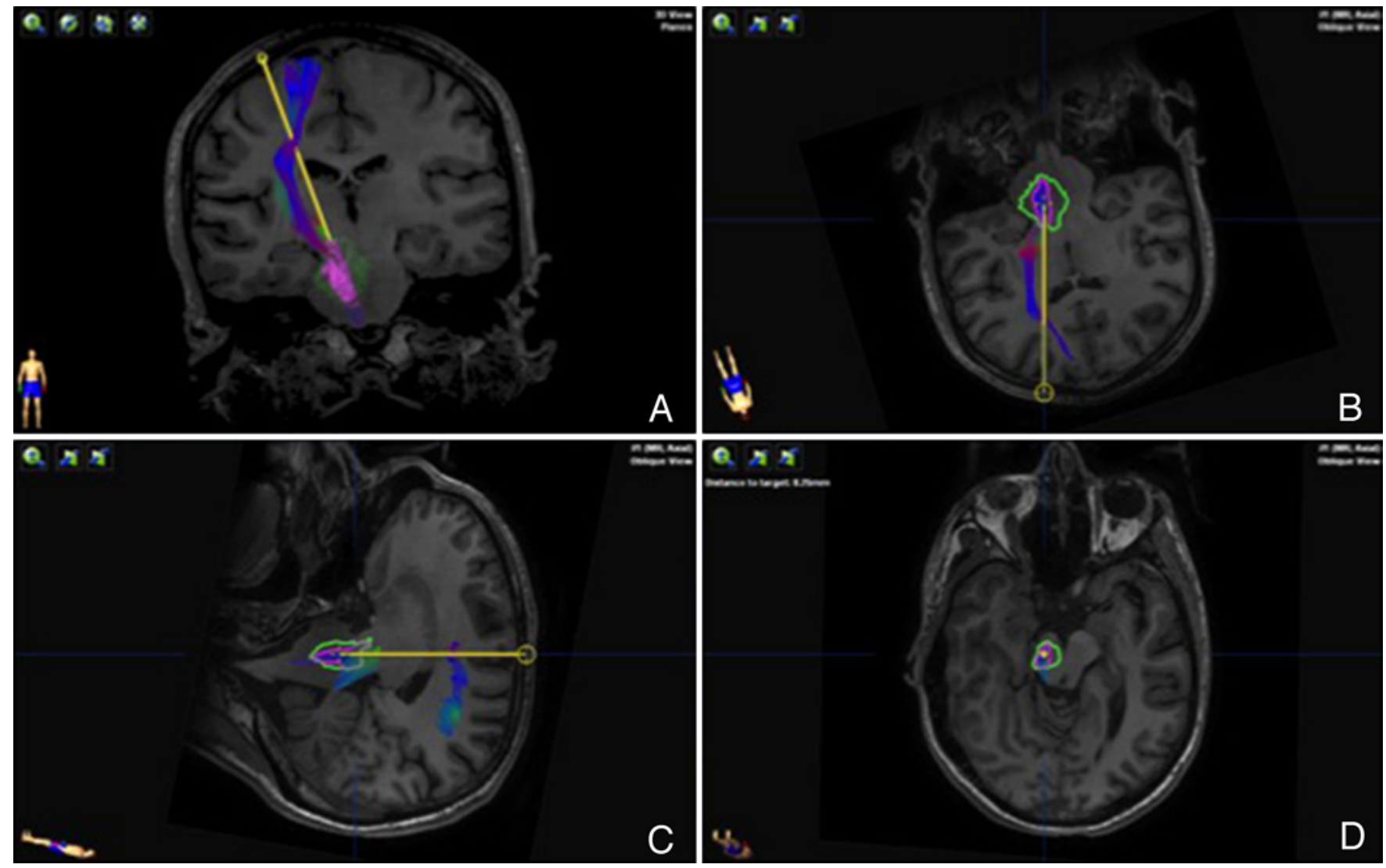

FIG. 2. Volumetric T1-weighted MRI demonstrating the catheter position (yellow) and right descending CST (multicolor; A), in line with the catheter (B and C) and probe view (D) for a patient undergoing right frontal placement of an infusion catheter. The overlap volume between the $3 \mathrm{D}$ fiber bundle in the pons (purple 3D object $[A]$, purple outline $[B-D]$ ) and the infusate distribution (green $3 D$ object $[\mathrm{A}]$, green outline $[\mathrm{B}-\mathrm{D}])$ is recorded.

on the highest dose level and had an overlap of $3.64 \mathrm{~cm}^{3}$ between Vd and CST.

Three patients $(3 / 9,33 \%)$ in the highest dose level experienced transient postinfusion deficits. Two of these were grade 2 , and one grade 3 , based on CTCAE version 4. Each of these patients experienced worsening of a baseline contralateral hemiparesis that resolved within 1 week. In the 6 patients without deficits after infusion, the mean number of catheter-CST intersections was 2.7, the mean length of intersection was $17.6 \mathrm{~mm}$, and the mean overlap between Vd and CST was $5.1 \mathrm{~cm}^{3}$ (range $0.8-10.8 \mathrm{~cm}^{3}$ ). In the 3 patients with postinfusion deficits, the mean number of catheter-CST intersections was 2, the mean length of intersection was $12.9 \mathrm{~mm}$, and the mean overlap between $\mathrm{Vd}$ and CST was $6.3 \mathrm{~cm}^{3}$ (range $3.5-10.3 \mathrm{~cm}^{3}$ ). Correlation analysis showed that in this group of patients, for number of intersections versus grade of neurological deficits, Spearman's $\rho=0.21(\mathrm{p}=0.60)$; for length of intersection versus grade of neurological deficits, Spearman's $\rho=-0.14$ $(\mathrm{p}=0.72)$; and for Vd-CST overlap volume versus grade of neurological deficits, Spearman's $\rho=-0.01(p=0.98)$.

\section{Discussion}

Direct delivery of therapeutics for neurooncological purposes has recently been introduced into early phase clinical trials for supratentorial glioma and intrinsic brainstem tumors. ${ }^{1-3,7-9,13,14,17}$ The use of CED for DIPG is of particular interest because of the current dearth of effective therapeutic strategies and reasonable volumetric compartment of this tumor relative to supratentorial gliomas; however, reaching brainstem targets with catheters has inherent risks. Surgical access routes for catheter insertion and distortion of corticofugal motor fibers with infusion are realistic concerns.

Studies of stereotactic biopsy approaches have shown that reaching the brainstem by transfrontal or transcerebellar corridors is safe with relatively low morbidity rates. ${ }^{4,5,10-12}$ Furthermore, early clinical studies of CED to brainstem targets have demonstrated a high degree of trajectory accuracy. ${ }^{3}$ In early case reports, tolerance to brainstem CED was not universally promising. Variance in surgical approaches, infusion volumes, therapeutic agent, and disease continuum limited meaningful interpretation of the earliest clinical application of this therapeutic strategy. Infusion rate has been shown to affect the rate of perioperative deficits in small series, ${ }^{1}$ and in animal models higher infusion volumes have been shown to alter normal anatomical relationships. ${ }^{16}$ Stereotactic biopsy with intra- 
TABLE 1. Characteristics and results for 30 patients with DIPG undergoing catheter placement into the brainstem

\begin{tabular}{lc}
\hline \multicolumn{1}{c}{ Characteristic } & Value (\%) \\
\hline Age, yrs & 7.6 \\
Mean & $3.2-18$ \\
Range & 18 \\
Sex & 12 \\
Male & \\
Female & 105.5 \\
Catheter trajectory length, mm & $92.7-121.6$ \\
Mean & 18.9 \\
Range & $0-44.2$ \\
\hline Length of CST intersection, mm & \\
Mean & 2.2 \\
Range & $0-3$ \\
\hline No. of CST intersections & $0(0.0)$ \\
Mean & $1(3.3)$ \\
\hline Range &
\end{tabular}

operative MR guidance and superimposed tractography to avoid transgression of these fibers has been described..$^{15}$ However, there has been no quantification of the interaction between catheter position and eloquent fibers, or of the effect of infusate distribution on these fibers.

We demonstrate that CED catheter placement frequently traverses the descending CSTs without causing clinical deficits when using a transfrontal approach. The one patient with a procedure-associated worsened facial palsy had an intersecting distance of $31.7 \mathrm{~mm}$ with 2 total intersections, on the higher end of the range for each within this cohort. However, due to the rarity of catheterassociated complications, it is not possible to directly correlate the length of catheter intersection with a higher risk of complications, although one might hypothesize that this could be the case. Additionally, the isolated nature of this particular patient's clinical deficit supports the notion that the facial paresis was attributable to distortion at the cranial nerve nucleus or lower motor nerve fiber level.

When evaluating overlap between distribution volume and CSTs, we found no correlation between overlap volumes and rates of postinfusion motor deficits. Furthermore, among those patients evaluated from the highest dose level, permanent deficit was nonexistent. The evaluation of overlap between distribution volume and corticofugal motor fibers as a predictor of postoperative complications is limited by the low number of patients who had recognized motor deficits. Furthermore, the small sample size and low frequency of complications preclude controlling for tumor burden and its influence on sensitivity of CST to transgression by either catheter or infusate distribution. We hypothesize that greater tumor burden or preexisting motor deficits could make patients more sensitive to perturbations caused by infusate distribution. The observation that all patients with transient deficits in this cohort experienced worsening of preexisting deficits sup-
TABLE 2. Characteristics and results for 9 patients with DIPG undergoing ${ }^{124} \mathrm{I}-8 \mathrm{H} 9$ infusion in the brainstem

\begin{tabular}{|c|c|}
\hline Characteristic & Value (\%) \\
\hline \multicolumn{2}{|l|}{ Age, yrs } \\
\hline Mean & 7.6 \\
\hline Range & $5.8-10.3$ \\
\hline \multicolumn{2}{|l|}{ Sex } \\
\hline Male & 5 \\
\hline Female & 4 \\
\hline \multicolumn{2}{|l|}{ Catheter trajectory length, $\mathrm{mm}$} \\
\hline Mean & 109.5 \\
\hline Range & $102.6-122.3$ \\
\hline \multicolumn{2}{|l|}{ Length of intersection, $\mathrm{mm}$} \\
\hline Mean & 16.0 \\
\hline Range & $0-34.6$ \\
\hline \multicolumn{2}{|l|}{ No. of intersections } \\
\hline Mean & 1.6 \\
\hline Range & $0-3$ \\
\hline \multicolumn{2}{|l|}{ Overlap between Vd and CST, $\mathrm{cm}^{3}$} \\
\hline \multicolumn{2}{|l|}{ Overall $(n=9)$} \\
\hline Mean & 5.5 \\
\hline Range & $0.8-10.8$ \\
\hline \multicolumn{2}{|l|}{ Without deficit $(n=6)$} \\
\hline Mean & 5.1 \\
\hline Range & $0.8-10.8$ \\
\hline \multicolumn{2}{|l|}{ With deficit $(n=3)$} \\
\hline Mean & 6.3 \\
\hline Range & $3.5-10.3$ \\
\hline Transient deficit after infusion & $3(33.3)$ \\
\hline Permanent deficit after infusion & $0(0)$ \\
\hline
\end{tabular}

ports this hypothesis, but this will need to be evaluated in a larger cohort.

This study has several limitations. First, the analysis of catheter intersection of CST is limited by the resolution of tractography and the thresholds set therein. We adhered to published protocols for CST tractography in patients with brainstem pathology. ${ }^{6}$ In a similar manner, it is important to note that the somatotopic organization of corticofugal fibers could affect the probability of a deficit when a catheter violates a particular area of the fiber bundle. We are unable to segment the fiber bundle with a level of precision that allows for a more detailed analysis of this point. Another limitation lies in the estimation of Vd using T2-weighted imaging. It is unknown how the altered $\mathrm{T} 2$-weighted signal compares with changes in actual drug or fluid dynamics. Subthreshold fluid changes might exist beyond the resolution of $\mathrm{T} 2$-weighted imaging.

\section{Conclusions}

Transgression of corticospinal fibers during catheter placement and by infusate distribution is well tolerated with a negligible rate of permanent neurological complications. This same encouraging result might not be transferable to other device design, guidance system, or surgical planning platforms. The tolerance profile of this 

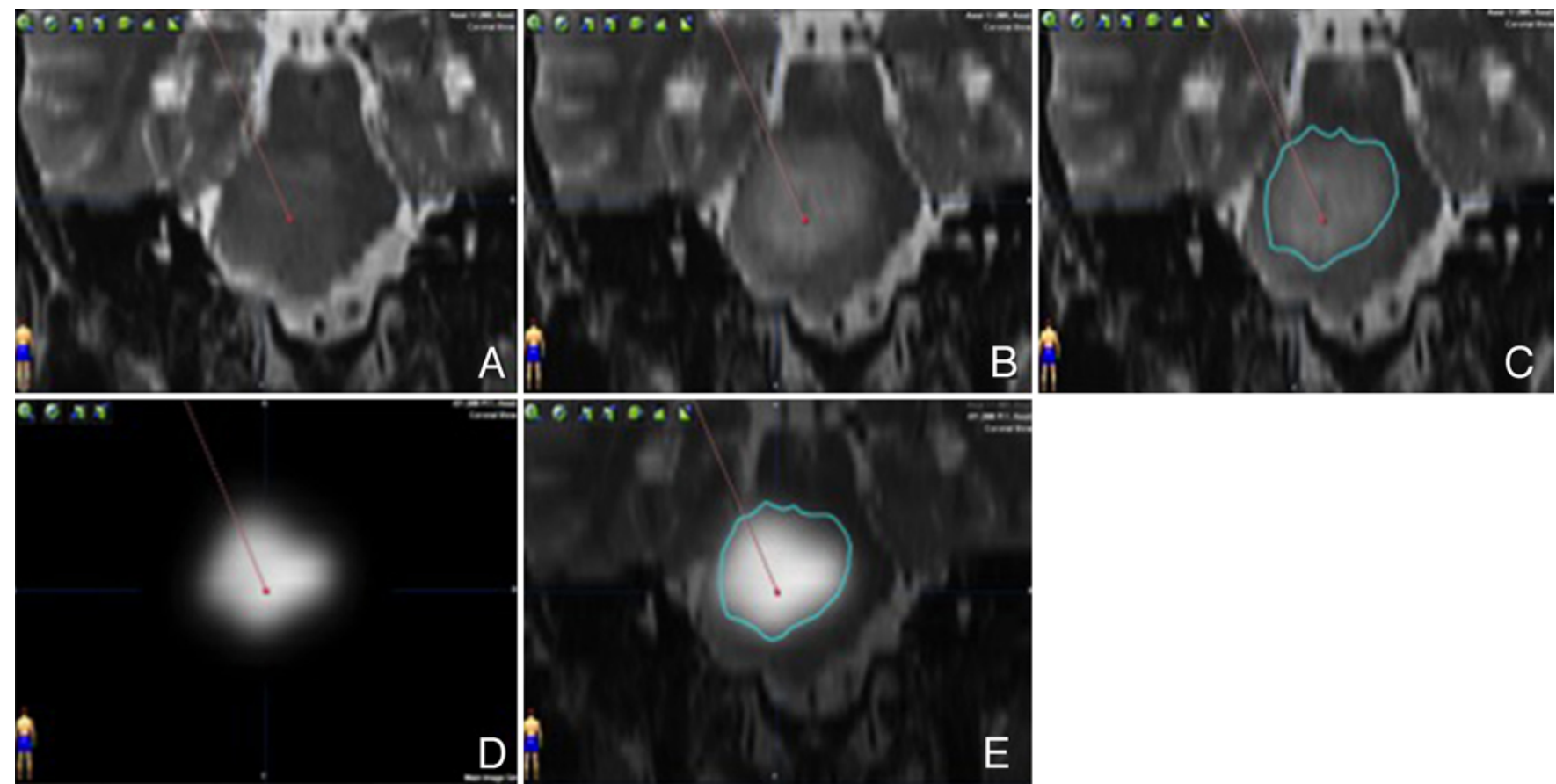

FIG. 3. Coronal reconstruction of T2-weighted images at baseline depicting the actual catheter position (red line) and preexisting T2-weighted hyperintensity consistent with an infiltrative pontine neoplasm at the infusion site (A). A coronal reconstruction of T2-weighted images post ${ }^{124} \mathrm{I}-8 \mathrm{H} 9$ infusion is shown (B). Increased T2-weighted signal surrounding the catheter tip is outlined in turquoise (Vd; C). A coronal PET image shows the distribution of ${ }^{124} \mathrm{I}-8 \mathrm{H} 9$ (D). A fused image demonstrates the congruence between the T2-weighted hyperintensity (turquoise outline) and PET image after infusion (E).

procedure is likely affected by infusion rate and individual agent tolerance, neither of which is evaluated as part of this study. Future studies with high-resolution segmentation of anatomical structures in the brainstem and larger numbers of patients could also be used to evaluate catheter position and infusate distribution in relation to cranial nerve nuclei or major sensory tracts, to further elucidate the mechanism of the tolerance.

\section{Acknowledgments}

This study was funded by: NIH grant no. P30-CA008748, the Dana Foundation Neuroimmunology Program, The Cure Starts Now, Solving Kids' Cancer, The Lyla Nsouli Foundation for Children's Brain Cancer Research, and Cookies for Kids' Cancer; contributions and donations from The Cristian Rivera Foundation, Battle for a Cure, Cole Foundation, Meryl \& Charles Witmer Charitable Foundation, and Tuesdays with Mitch Charitable Foundation; and intramural funding from Memorial Sloan Kettering Cancer Center (Pediatric Neurosurgery Fund, Center for Experimental Therapeutics grant, Fred's Team and Perry's Promise).

\section{References}

1. Anderson RC, Kennedy B, Yanes CL, Garvin J, Needle M, Canoll P, et al: Convection-enhanced delivery of topotecan into diffuse intrinsic brainstem tumors in children. J Neurosurg Pediatr 11:289-295, 2013

2. Barua NU, Lowis SP, Woolley M, O'Sullivan S, Harrison R, Gill SS: Robot-guided convection-enhanced delivery of carboplatin for advanced brainstem glioma. Acta Neurochir (Wien) 155:1459-1465, 2013
3. Chittiboina P, Heiss JD, Lonser RR: Accuracy of direct magnetic resonance imaging-guided placement of drug infusion cannulae. J Neurosurg 122:1173-1179, 2015

4. Dellaretti M, Reyns N, Touzet G, Dubois F, Gusmão S, Pereira JL, et al: Stereotactic biopsy for brainstem tumors: comparison of transcerebellar with transfrontal approach. Stereotact Funct Neurosurg 90:79-83, 2012

5. Hamisch C, Kickingereder P, Fischer M, Simon T, Ruge MI: Update on the diagnostic value and safety of stereotactic biopsy for pediatric brainstem tumors: a systematic review and meta-analysis of 735 cases. J Neurosurg Pediatr 20:261268, 2017

6. Helton KJ, Weeks JK, Phillips NS, Zou P, Kun LE, Khan RB, et al: Diffusion tensor imaging of brainstem tumors: axonal degeneration of motor and sensory tracts. J Neurosurg Pediatr 1:270-276, 2008

7. Kunwar S, Chang S, Westphal M, Vogelbaum M, Sampson J, Barnett G, et al: Phase III randomized trial of CED of IL13-PE38QQR vs Gliadel wafers for recurrent glioblastoma. Neuro Oncol 12:871-881, 2010

8. Kunwar S, Prados MD, Chang SM, Berger MS, Lang FF, Piepmeier JM, et al: Direct intracerebral delivery of cintredekin besudotox (IL13-PE38QQR) in recurrent malignant glioma: a report by the Cintredekin Besudotox Intraparenchymal Study Group. J Clin Oncol 25:837-844, 2007

9. Lonser RR, Warren KE, Butman JA, Quezado Z, Robison RA, Walbridge $S$, et al: Real-time image-guided direct convective perfusion of intrinsic brainstem lesions. Technical note. J Neurosurg 107:190-197, 2007

10. Pincus DW, Richter EO, Yachnis AT, Bennett J, Bhatti MT, Smith A: Brainstem stereotactic biopsy sampling in children. J Neurosurg 104 (2 Suppl):108-114, 2006

11. Quick-Weller J, Lescher S, Bruder M, Dinc N, Behmanesh B, Seifert V, et al: Stereotactic biopsy of brainstem lesions: 
21 years experiences of a single center. $\mathbf{J}$ Neurooncol 129:243-250, 2016

12. Roujeau T, Machado G, Garnett MR, Miquel C, Puget S, Geoerger B, et al: Stereotactic biopsy of diffuse pontine lesions in children. J Neurosurg 107 (1 Suppl):1-4, 2007

13. Sampson JH, Akabani G, Archer GE, Berger MS, Coleman $\mathrm{RE}$, Friedman AH, et al: Intracerebral infusion of an EGFRtargeted toxin in recurrent malignant brain tumors. Neuro Oncol 10:320-329, 2008

14. Sampson JH, Archer G, Pedain C, Wembacher-Schröder E, Westphal M, Kunwar S, et al: Poor drug distribution as a possible explanation for the results of the PRECISE trial. J Neurosurg 113:301-309, 2010

15. Sun X, Chen Z, Yang S, Zhang J, Yue S, Wang Z, et al: Role of high-field intraoperative magnetic resonance imaging on a multi-image fusion-guided stereotactic biopsy of the basal ganglia: a case report. Oncol Lett 9:223-226, 2015

16. Valles F, Fiandaca MS, Bringas J, Dickinson P, LeCouteur R, Higgins R, et al: Anatomic compression caused by highvolume convection-enhanced delivery to the brain. Neurosurgery 65:579-586, 2009

17. Vogelbaum MA, Sampson JH, Kunwar S, Chang SM, Shaffrey M, Asher AL, et al: Convection-enhanced delivery of cintredekin besudotox (interleukin-13-PE38QQR) followed by radiation therapy with and without temozolomide in newly diagnosed malignant gliomas: phase 1 study of final safety results. Neurosurgery 61:1031-1038, 2007

\section{Disclosures}

Dr. Souweidane reports being a consultant to Aesculap.

\section{Author Contributions}

Conception and design: Souweidane. Acquisition of data: Morgenstern, Zhou, Wembacher-Schröder, Cina, Tsiouris. Analysis and interpretation of data: all authors. Drafting the article: Morgenstern, Zhou, Wembacher-Schröder. Critically revising the article: Morgenstern, Zhou, Wembacher-Schröder, Tsiouris, Souweidane. Reviewed submitted version of manuscript: all authors. Approved the final version of the manuscript on behalf of all authors: Morgenstern. Statistical analysis: Zhou. Study supervision: Souweidane.

\section{Correspondence}

Peter F. Morgenstern: NewYork-Presbyterian Hospital, Weill Cornell Medical Center, New York, NY.pfmorgenstern@gmail.com. 